\title{
About the control of the electric elastic resistance of a single molecule
}

\author{
Christian Joachim \\ Molecular Electronic Group, CEMES-LOE/CNRS, 29 rue Jeanne Marvig, B.P. 4347, 31055 Toulouse \\ cedex, France
}

(Received October 01, 1990; accepted January 12, 1991)

\begin{abstract}
Résumé. - Le contrôle de la résistance électrique élastique d'une seule molécule est discuté à partir de la définition de cette résistance basée sur la formule de Landauer. Cette définition est utilisée pour distinguer entre les effets de constriction et les effets intramoléculaires. Des exemples de commutateurs moléculaires sont présentés, l'un utilisant un effet mécanique, l'autre un effet électronique. Le besoin d'une microscopie à résolution atomique est souligné dans le but de fabriquer et de brancher électriquement ces commutateurs moléculaires.
\end{abstract}

\begin{abstract}
The control of the elastic electric resistance of a single molecule is discussed using a definition of molecular elastic resistance based on the Landauer formula. This definition is discussed in the prospect to distinguish between constriction effects and intramolecular effects. Examples of molecular switches are given, one using a mechanical effect, the other an electronic effect. The need for atomic resolved microscopy is emphasized to fabricate and wire such molecular switches.
\end{abstract}

\section{Introduction.}

For an electrode surface area less than $100 \mu \mathrm{m}^{2}$, the current passing through a metal-vacuummetal tunnel junction with a tunneling gap longer than $1 \mathrm{~nm}$ is smaller than the $\mu \mathrm{A}$ at a bias voltage lower than $100 \mathrm{mV}$ [1]. To get a higher current over the same gap, one needs to fill up this gap with an inorganic (organic) material. This has been first experimented by Fisher and Giaever on $\mathrm{Al}-\mathrm{Al}_{2} \mathrm{O}_{3}-\mathrm{Al}$ junctions [2], by Esaki on abrupt semiconductor diodes [3] and more recently by Kuhn and co-workers on Al-LB-Al junctions with LB a Langmuir Blodgett organic film [4]. The effect of these materials is to lower the apparent tunnel barrier height experimented by the electrons [5]. Therefore, the current intensity can reach at least the $\mu \mathrm{A}$ range for the same surface area of the electrodes.

With the advance of nanolithography techniques and also with the invention of the scanning tunneling microscope (STM) such a tunnel experiment can now ne done at a much lower scale. If one has a $1 \mathrm{~nm}$ vacuum gap between two nanoscopic $10 \mathrm{~nm}$ in wiuth metallic wires, the tunneling 
current through this junction will be smaller than with macroscopic electrodes due to the large reduction of the electrodes surface area in this case. But like in the Giaever or Kuhn experiments, a droplet of material can be embedded in the vacuum gap between the two nanoscopic wires to increase the tunneling current intensity. More interesting is to "short circuit" the vacuum tunneling gap by a small amount of molecules (idealy one) well ordered between the two nanoscopic wires [6].

However, one may consider that nothing useful can happen inside a small object like a single molecule embedded between two metallic wires. But for more than 30 years, physicists and chemists have tried to understand the wealth of the internal world of a single molecule, which seems too poor in degrees of freedom at a first glance. We have learn how electrons (holes) added to a single molecule can move through it [7], how energy provided to a molecule can be totally reemitted or shared between the electron and the nuclei populations [8]. This is the richness of the intramolecular world which has lead to the expectation than one day a single molecule will be used in electronic devices in place of the solid state transistor [9]. The metal-single molecule-metal junction is one approach to this expectation $[6,10]$.

This approach will be described in this contribution. In section 2 , the concept of resistance of a single molecule is discussed. In section 3, some possibilities to control the resistance of the molecule by an external perturbation are described. In section 4 , the need for a local characterization technique such as the STM or the AFM (Atomic Force Microscope) is emphasized. This section 4 makes the connection with the overall topic of this special issue of Microscopy, Microanalysis and Microstructures. It will clarify the need for powerful characterization techniques of inorganic surfaces as soon as the material experimented on theses surfaces is nanoscopic in size.

\section{The elastic resistance of a single molecule.}

Let us consider the electrical circuit figure 1. As already discussed [11], nanofabrication techniques are in the way of providing the metallic wiring needed to fabricate such a circuit. However, the chemisorption of a single molecule in parallel on the nanoscopic tunneling gap is truly a delicate problem [11]. But it will not be discussed here. Therefore, the "gedanken" circuit in figure 1 is considered in the following as a working example to get more insight on the physics involved at this scale.

As already noticed by Frenkel in the early 30's [12], the current $(I)$ - voltage $(V)$ characteristic of a tunneling junction is truly non-linear. But at low bias voltage (typicaly lower than $100 \mathrm{mV}$ ), only the linear part of the $I=f(V)$ characteristic can be kept. For the circuit figure 1, such an approximation will also be used. First, because it is very instructive to understand how the resistance of a metal-single molecule-metal junction can be controlled without involving nonlinear effect. Secondly, because up to now, no developpement of the $I=f(V)$ function in series of the bias voltage for a given molecule has been obtained. Notice on the contrary that to analyse the optical response of a molecule, the expression of the polarization in series of the local electric field has been very well worked out since the seminal work of Bloembergen and co-workers [13].

Our next hypothesis to define the molecule electrical resistance in the circuit figure 1 concerns how electrons find their way from the macroscopic wires A and B to the molecule through the mesoscopic wires $C$ and $D$. Truly, the temperature of these wires must be as low as $10 \mathrm{~K}$. In that case, the electron mean free path inside the wires is of the order of the micron [14]. Therefore, for well crystalline metallic wires, localization phenomena will not affect the experiment and the electrons will reach the molecule without phase destruction.

Due to the bias voltage $V$, the chemical potential $\mu_{\mathrm{A}}$ of wire $\mathrm{A}$ is different from the one $\mu_{\mathrm{B}}$ of wire B. In the case, a net electronic current will flow from the wire with the higher chemical 


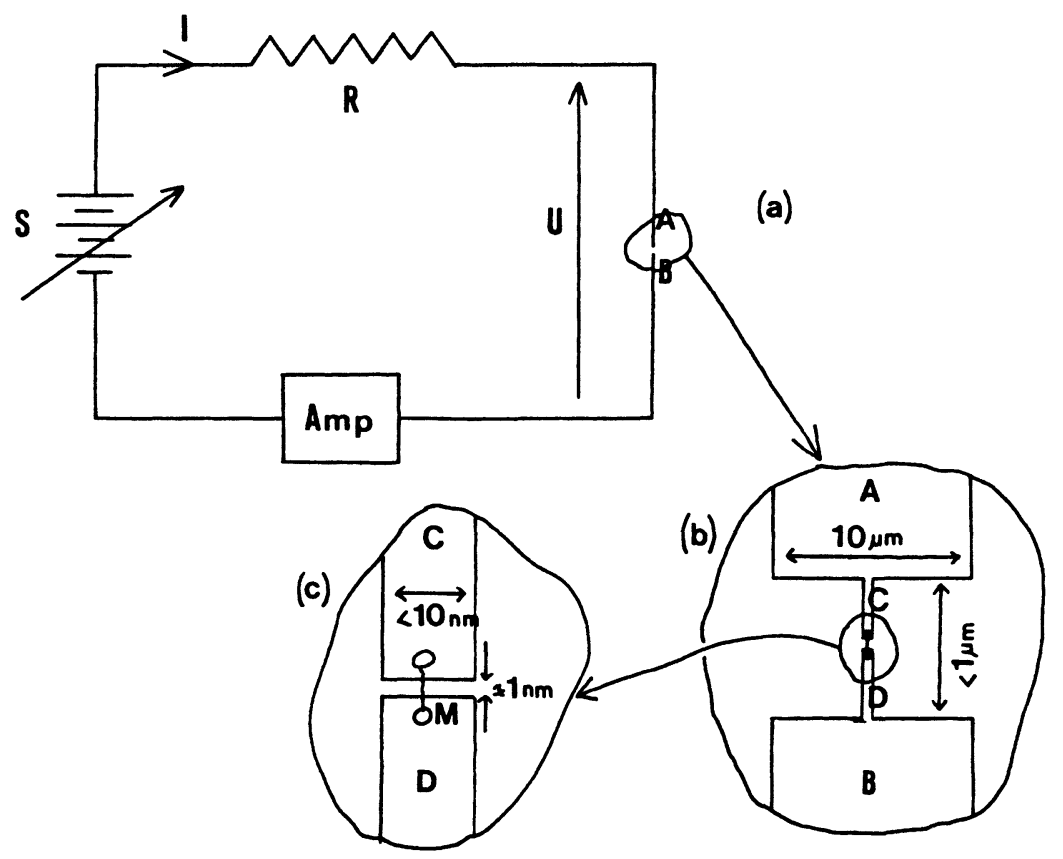

Fig. 1. - Electrical circuit proposed to measure the electrical resistance $R_{\text {mol }}$ of a single molecule. (a) is the macroscopic scale view of this circuit, (b) a mesoscopic scale view and (c) the nanoscopic view.

potential to the wire with the lower one. At low bias voltage, $\mu_{\mathrm{A}}-\mu_{\mathrm{B}}=\mathrm{eV}$ is small too and the overall tunnel current intensity $I$ in the circuit is proportional to $\mu_{\mathrm{A}}-\mu_{\mathrm{B}}$ [15]:

$$
I=e^{-1} G\left(\mu_{\mathrm{A}}-\mu_{\mathrm{B}}\right)
$$

Before discuting the meaning of $G$, notice that (1) is only valid at low temperature to be sure that no inelastic scattering occurs. Moreover, with inelastic scattering events, (1) cannot be used because tunneling probabilities from wire $A$ to wire $B$ and from wire $B$ to wire $A$ are different in this case [15]: the time reversal property is lost due to the dissipative character of an inelastic scattering process.

$G$ is the conductance of the overall metallic wire C, D and of the molecule. Its expression is given by calculating the transmission coefficients $\left|t_{\alpha \alpha^{\prime}}\right|^{2}$ of an electron coming from an electron band $\alpha$ of wire A and tunneling to an electron band $\alpha^{\prime}$ of wire $\mathrm{B}[16]$ :

$$
G=\frac{e^{2}}{\pi \hbar} \sum_{\alpha, \alpha^{\prime}}\left|t_{\alpha \alpha^{\prime}}\left(E_{\mathrm{F}}\right)\right|^{2}
$$

In (2), $t_{\alpha \alpha^{\prime}}(E)$ is taken at the Fermi Level $E_{\mathrm{F}}$ of the wires because of the linear approximation leading to (1). Therefore, $G$ is an elastic conductance i.e. a characterization of the way a flux of ballistic electrons coming from A (or B) will be transmitted by the mesoscopic part of the circuit figure 1. As in any electrical circuit, the energy dissipation occurs according to the intensity of the current. But this dissipation is located in first approximation in the wires A and B where inelastic electrons-phonons scattering events occurs. In these wires, this will require a few microns to 
become effective. At low temperature, the mesoscopic part of the circuit cannot be considered as a dissipative element and the Joule law $P=G^{-1} I^{2}$ doesn't applied here.

Unfortunately, $\Omega$ is not exactly the resistance of the molecule embedded in the circuit figure 1 . This is due to the fact that the size reduction from $A$ to $C$ and the enlargement from $D$ to $B$ introduced electron wave reflections, the so-called constriction resistance, which depend on the current passing through the circuit [17]. In principe, one can evaluate this resistance by measuring the voltage drop accross the molecule. This requires for example two probes connected to the mesoscopic wire $\mathrm{C}$ and $\mathrm{D}$ as close as possible to the molecule. But such an invasive measurement completely modifies the electrical circuit close to the molecule. Therefore, the overall tunneling current will change too. The same measurement problem have been discussed in the context of measuring chemical potential drop along a mesoscopic wire with non-invasive probe [18]. Without entering in the debate, one can notice that even the definition of a chemical potential for the wire $\mathrm{C}$ and for the wire $\mathrm{D}$ is not clear. Since there is no inelastic scattering event, the electrons passing through the wires $\mathrm{C}$ and $\mathrm{D}$ are out of equilibrium with the lattice. Therefore, in principle, it is not possible to define a chemical potential. Nevertheless, one can play with the electron distribution functions and define a quasichemical potential for each wire $\mathrm{C}$ and $\mathrm{D}$. This can be done by considering that in the steady state region, the average number of electrons is constant in the wires $\mathrm{C}$ and $\mathrm{D}$ [9]. As we have demonstrated [20] this is equivalent to break the non-separability of the "constriction-mesoscopic wire -single molecule-mesoscopic wire-constriction" quantum system and to use a quasi-classical description of this system. In this approximation, the overall resistance $R=G^{-1}$ of the system is the sum of two effective constriction resistances $R_{\mathrm{c}}$ with the resistance of the molecule $R_{\mathrm{mol}}$ :

$$
R=2 R_{\mathrm{c}}+R_{\mathrm{mol}}
$$

But $R_{\mathrm{c}}$ is a function of the number of channel available to the electron in the mesoscopic wire C and $\mathrm{D}[21]$ :

$$
R_{\mathrm{c}}=\frac{\pi \hbar}{2 e^{2} N_{\mathrm{c}}}
$$

with $N_{\mathrm{c}}$ the number of active channel in the wires C and D at the Fermi level of the wires A and B. In this semi-classical approximation, the apparent resistance of the molecule in the circuit figure 1 is given by combining (3) and (4) with $R=G^{-1}$ :

$$
R_{\mathrm{mol}}=\frac{\pi \hbar}{e^{2} N_{\mathrm{c}}} \frac{1-T_{\mathrm{eff}}}{T_{\mathrm{eff}}}
$$

with $T_{\text {eff }}$ given from (2) by:

$$
T_{\text {eff }}=\frac{\pi \hbar}{e^{2} N_{c}}
$$

Truly, (5) is not exact. The approximation is quasi-classical because (4) can only be calculated at the quantum level and (3) is derived from the classic Ohm's law. More important, $R_{\text {mol }}$ explicity depends on the mesoscopic wires used to connect this molecule. When $N_{\mathrm{c}}$ increases, $\mu_{\mathrm{C}}$ becomes close to $\mu_{\mathrm{A}}$ and $\mu_{\mathrm{D}}$ to $\mu_{\mathrm{B}}$. This means that $\mu_{\mathrm{C}}-\mu_{\mathrm{D}}$ not only depends on the molecule electrical properties but also on the size and nature of the metal chosen for the mesoscopic wire C and D. This dependance is characteristic, even in a quasi-classical approximation, of the relation between the measurement and the quantum system under study. Moreover (5) provides a way to evaluate, for a given wiring system, how a single molecule controls the current intensity in the circuit figure 1. 


\section{Control of the elastic resistance of a single molecule.}

In the linear regime, without inelastic scattering, the elastic resistance $R_{\text {mol }}(5)$ relies only on $T_{\text {eff }}$ $\left(E_{\mathrm{F}}\right)$. Following (2), $T_{\mathrm{eff}}(E)$ is the trace of a $t t^{+}$matrix where $t$ is the transmission part of the scattering matrix describing the scattering of the electrons on the metal-molecule-metal junction. $T_{\text {eff }}(E)$ is a function of the shape and position of the band structure of the wires A and B and of the electronic structure of the mesoscopic wires $\mathrm{C}$ and $\mathrm{D}$. But $T_{\text {eff }}(E)$ is also dependent of the coupling $C_{\text {eff }}(E)$ between the wires $\mathrm{A}$ and B Bloch states through the metal-molecule- metal junction $[22,23]$. Therefore, to play with $R_{\mathrm{mol}}$, it is possible to tune the electronic structure of the wires or the coupling through the molecule.

Moreover, a lot of expertises have been accumulated on the control of through bridge electronic effective coupling in mixed valence compounds [10]. Then it is worthwhile to use these results to design active molecules like molecular wires or molecular switches.

Before presenting examples of such molecules, an important point to discuss is the definition of $C_{\text {eff }}(E)$ in our context. Let us take an electronic state $\left|\psi_{\mathrm{C}}\right\rangle$ of the wire C when no molecules are chemisorbed on the tunneling gap and one electronic state $\left|\psi_{\mathrm{D}}\right\rangle$ of the wire D with $\left\langle\psi_{\mathrm{C}} \mid \psi_{\mathrm{D}}\right\rangle=0$ and $\left\langle\psi_{\mathrm{C}}|H| \psi_{\mathrm{D}}\right\rangle=0$ for the tunneling gap length chosen. Theses states can be pure electronic Bloch states or a mixing of such states. When a molecule is chemisorbed, the interaction between $\left|\psi_{\mathrm{C}}\right\rangle$ and $\left|\psi_{\mathrm{D}}\right\rangle$ is established via intermediate molecular states $\left|\varphi_{\text {mol }}\right\rangle$ such that $\left\langle\psi_{\mathrm{C}}|H| \varphi_{\text {mol }}\right\rangle$ $\left\langle\varphi_{\text {mol }}|H| \psi_{\mathrm{D}}\right\rangle \neq 0$. Then, the electronic coupling between $\left|\psi_{\mathrm{C}}\right\rangle$ and $\left|\psi_{\mathrm{D}}\right\rangle$ through the molecule must be evaluated using a model Hamiltonian $H_{\text {eff }}$ which sums up all the possible interaction channels via the molecule. It is a model Hamiltonian since Trace $\left(H_{\text {eff }}\right)<$ Trace $(H)$. In general, $H_{\text {eff }}$ comes from a transformation $U$ of $H: H_{\text {eff }}=U^{-1} H U$ followed by a projection on a model space [23]. Therefore by definition:

$$
H_{\mathrm{eff}}(E)_{\mathrm{CD}}=\left\langle\psi_{\mathrm{C}}\left|H_{\mathrm{eff}}\right| \psi_{\mathrm{D}}\right\rangle=\left\langle\varphi_{\mathrm{C}}|H| \varphi_{\mathrm{D}}\right\rangle
$$

with $\left|\varphi_{\mathrm{C}}\right\rangle=\left(U^{-1}\right)^{+}\left|\psi_{\mathrm{C}}\right\rangle$ and $\left|\varphi_{\mathrm{D}}\right\rangle=U\left|\psi_{\mathrm{D}}\right\rangle$ the so-called diabatic states of the states $\left|\psi_{\mathrm{C}}\right\rangle$ and $\left|\psi_{\mathrm{D}}\right\rangle$. These states are used in the following to propose active molecules.

3.1 Molecular wires. - By definition, a molecular wire is a molecule which leads to a low $R_{\text {mol }}$ for a length as long as possible. Clearly, most of the organic molecules have a gap at the Fermi level of the metal used for the wires. Therefore, the tunneling process through such a molecule will be non resonant and $T_{\text {eff }}\left(E_{\mathrm{F}}\right)$ will decrease rapidly as a function of the length of the molecule [22]. Since a saturated molecule is usualy characterized by a very large HOMO-LUMO gap, a molecular wire must be a conjugated molecule. But it is well known that in most long conjugate organic molecules dimerization of the repeat unit occurs (the so called "Peierls distorsion"). This leads to an important decrease of $C_{\mathrm{eff}}\left(E_{\mathrm{F}}\right)$ as a function of the chain length [24]. Therefore, a molecular wire will get a high resistance compared to a metal chain of the same length.

Contrary to the case of mixed valence compounds [24], the optimization of a molecular wire has not been worked out. We have done some preliminary calculation on alkanes embedded between 2 nanoscopic gold wires [22] and some experiments using a STM on the same molecule [25]. But the small resistance found is still under debat [26]. The design of a good molecular wire is therefore an open problem, even if one can use the extensive work which has been devoted to organic and metallo-organic conducting polymers.

3.2 Molecular switches. - A molecular switch can be defined as a single molecule which under an external perturbation (light absorption, electric field) produces a drastic change of the current intensity in the circuit figure 1 . Following our knowledge on $C_{\mathrm{eff}}(E)$, this means in the 
linear approximation that for $E=E_{\mathrm{F}}$ (i.e. at low bias voltage) $C_{\mathrm{eff}}(E)$ must present a large variation as a function of the external perturbation chosen. Some simple rules based on the overlap of the diabatic wave function have been used for mixed valence complexes to design such active molecules [10]. For a metal-single molecule- metal junction, our guideline is also to play with the overlap. But before providing some examples, notice that the design of molecular switches based on the control of $C_{\text {eff }}(E)$ does not take into account non-linear effects. These effects appear when, for example, a large bias voltage is used in such a way that $E_{\mathrm{F}}+\mathrm{eV}$ crosses a molecular electronic level [6]. Only the control of $(\mathrm{d} I / \mathrm{d} V)_{V=0}$ is considered here.

They are various ways to control the overlap between diabatic wave functions with the constraint that the active molecule is attached to the two mesoscopic wires. They are summarized figure 2. The first possibility is to do "molecular mechanics" by moving a part of the molecule to make the two diabatic tails orthogonal or far appart. The second possibility is to do "molecular electronics" by directing the tails in opposite direction using donor (or acceptor) group or by forcing these tails to interfere. One example of each is presented in the following.
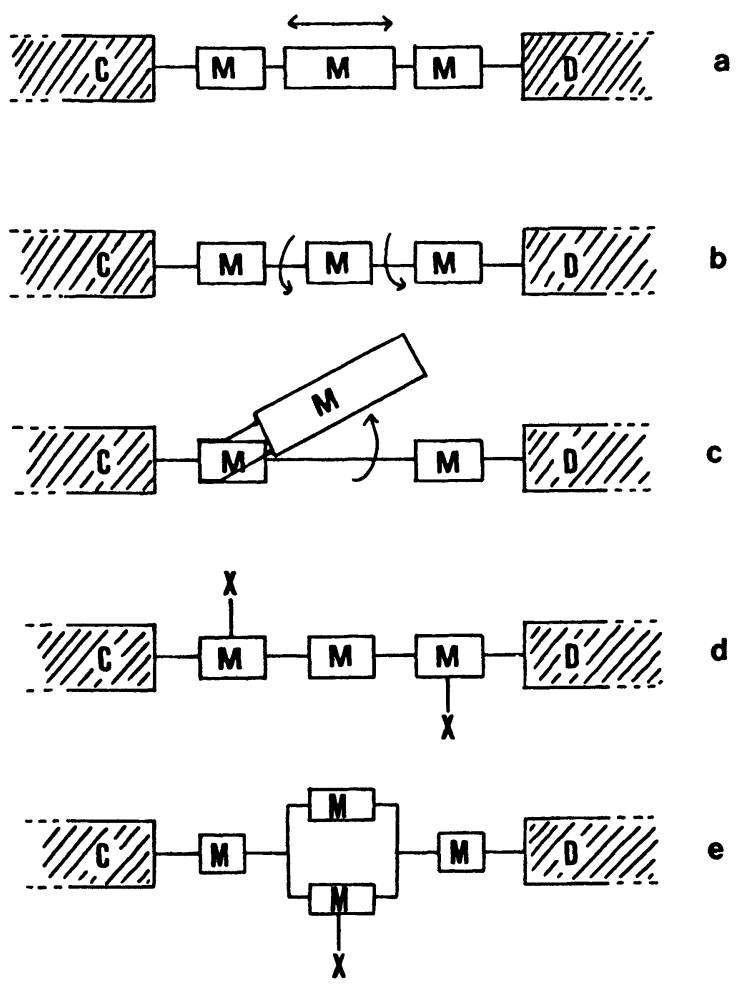

Fig. 2. - Differents startegies to control $R_{\mathrm{mol}}$. (2a), (2b) and (2c) are dealing with a conformational control of the diabatic wave funtion overlap and (2d) and (2e) to an electronic control of such an overlap. $\mathrm{X}$ is for a donor (or an acceptor) group.

The "mechanics" refers for example to conformation change of one part of the molecule by light absorption. One interesting effect in this context is the TICT effect i.e. the possibility of some non rigid donor acceptor (D-A) molecules to be twisted in the excited state [27]. This comes from the fact that in some $D-A$ molecules the $(D-A)^{*}$ state crosses a $D^{+}-A^{-}$state. The molecule 
embedded between 2 gold wires presented figure 3 has been designed using two such molecule assembled together.

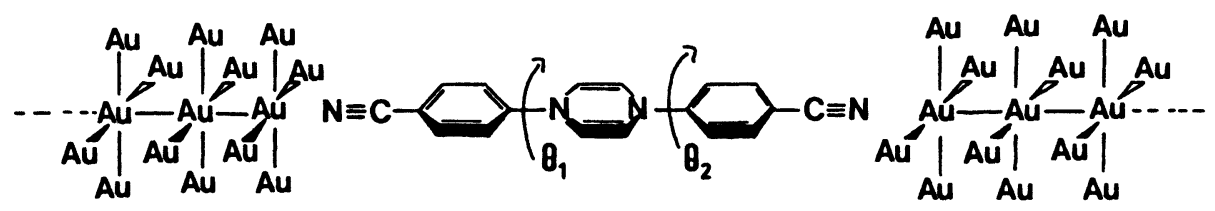

Fig. 3. - A model TICT molecule connected to two nanoscopic gold wires with a section of 5 atoms. This molecule is the $\mathrm{N}-\mathrm{N}^{\prime}$ Bis (4 cynanophenyl).- dihydropyrazine, a conjugated version of the TICT molecule studied in [27].

The variation of $T_{\text {eff }}(E)$ as a function of the rotation angle $\theta=\theta_{1}=\theta_{2}$ of the central part of this molecule is presented figure 5 for $\theta=0^{\circ}$ and $\theta=90^{\circ}$ only for the channels of symmetry $\pi$ of the gold wire [28]. It is clear that resonant molecular levels are shifted after a $\theta=0$ to $\theta=90^{\circ}$ rotation. This means that upon rotation, the $\pi-\pi$ coupling of the central part of the molecule with the two others is suppressed at $\theta=90^{\circ}$. Notice also that only the $\mathrm{C}_{3}$ channel gets a decreasing $T_{\text {eff }}(E)$. The two others $\pi$ channels have raised their $T_{\text {eff }}(E)$ when $\theta$ goes to $90^{\circ}$. This can be explained by a through $\sigma$ coupling via the $\sigma$ molecular orbitals of the central part which is activated at $\theta=90^{\circ}$. This example is characteristic of the difficulties we are facing to design a molecular switch: some coupling can be deactivated after a conformation change but other generally become active.

For the second possibility (the "electronics") to control diabatic tails overlap, it is interesting to consider the example of a benzene ring embedded in a polyacetylene chain as presented figure 5. The polyacetylene is there to model the wires band structure needed to send Bloch electron waves through the benzene ring. $T_{\text {eff }}(E)$ through this benzene ring is presented figure 5 . It is clear that the way the polyacetylene chains are connected to the benzene controls $T_{\text {eff }}(E)$. This comes from the fact that Bloch waves interfere through the ring. If one branch of the ring is longer than the other, the phase of the diabatic tails through this branch will be different from the other branch. A destructive interference between the two parts of the tails will lead to a low $T_{\text {eff }}(E)$ as shown figure 5. This open fascinating possibilities to control $T_{\text {eff }}(E)$ and therefore $R_{\text {mol }}$ using for example the ability of donor-acceptor groups to shift the energy position of the destructive interference.

\section{The need for a local characterization.}

The two major problems in the fabrication of the circuit presented figure 1 are first the characterization of the mesoscopic wires $C$ and $D$ and secondly the recognition of the chemisorbed molecule. For the first one, the quality of the wire metal and the respect of the wire dimension along its length are both important. As demonstrated section 2, the reason is the sensitivity of $R_{\text {mol }}$ to the electronic structure of the wires $\mathrm{C}$ and $\mathrm{D}$ via the tunneling coefficients $t_{\alpha \alpha^{\prime}}(E)$. This means that a $t_{\alpha \alpha^{\prime}}(E)$ can be modified very easily by a small variation in the lateral dimension of the wire. For that surpose, local probe microscopy like STM and AFM are needed to check with an atomic resolution the regularity of the lateral dimension of the wires [29]. Three contributions in this special issue are dealing with STM and AFM works. It is therefore not needed here to decribe how a STM or an AFM works. Moreover, this characterization will no be complete without 

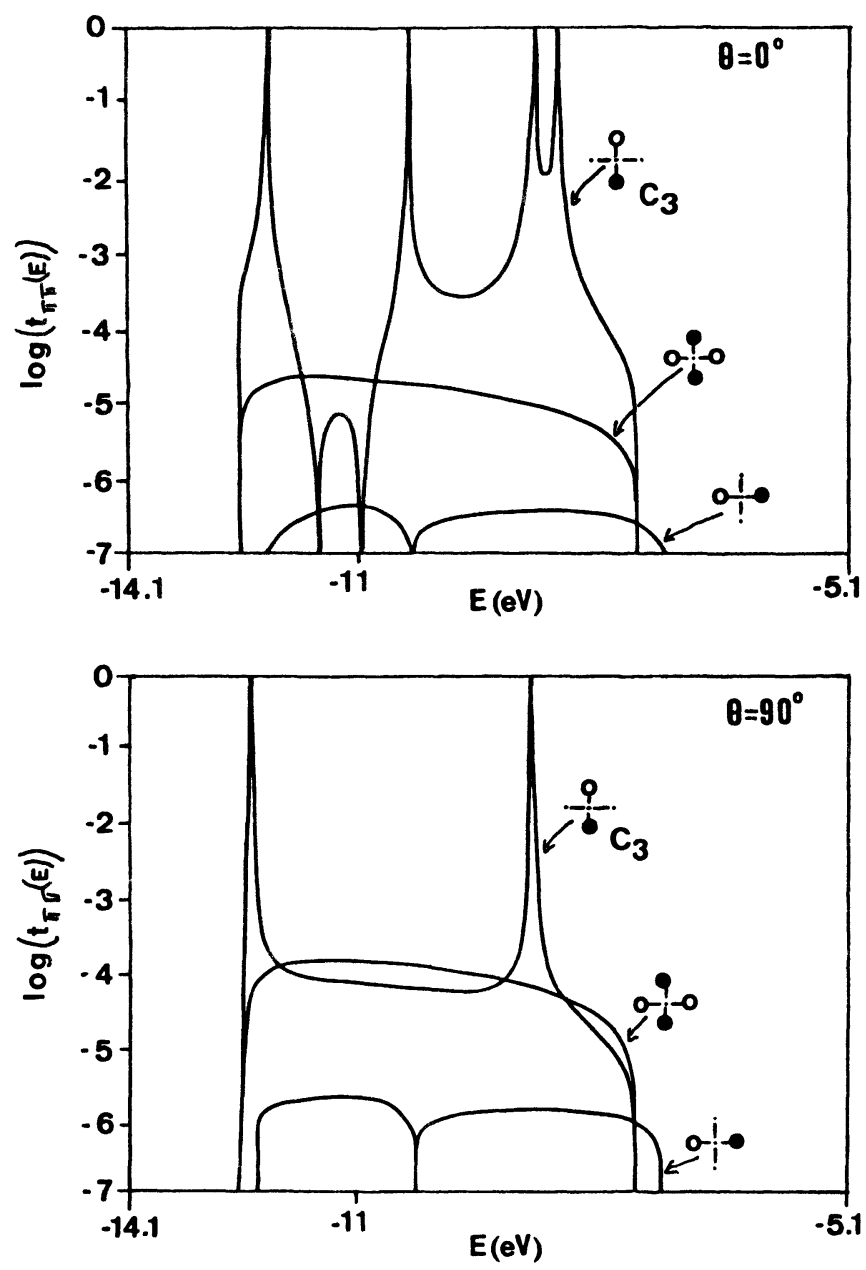

Fig. 4. - Transmission coefficients through the molecule figure 3 from the 3 channels of symmetry $\pi$ of the left gold wire to the same $\pi$ channels of the right gold wire. Only $6 \mathrm{~s}$ orbitals have been considered for Au atoms in this calculation. This lead to 5 propagative channels, 3 with $\pi$ symmetry [22].

an analysis in depth of the internal structure of these wires. In that prospect Transmission Electron Microscopies are going to be very useful to give for example the grain internal structure of the mesoscopic wires. This knowledge is important to optimize annealing processes used to avoid grain boundaries in these wires. Like lateral dimension variation along the wires, grain structures introduce elastic and inelastic scattering events in the mesoscopic wires. This will perturbe the measurement of $R_{\mathrm{mol}}$.

Concerning the recognition of the chemisorbed molecule, the problem is not only to use an instrument able to produce an atomic resolved image of the surface but also to recognize the species absorbed on the surface. This has always been a problem in microscopy in general and this problem is alsc present STM and AFM microscopies. For example the usual interpretation with STM is that an STM image depends on the density of states of the surface for $E=E_{\mathrm{F}}$ [30]. This work quite well tor semi-conductor surfaces like Si, GaAs but not totally with metal surface 

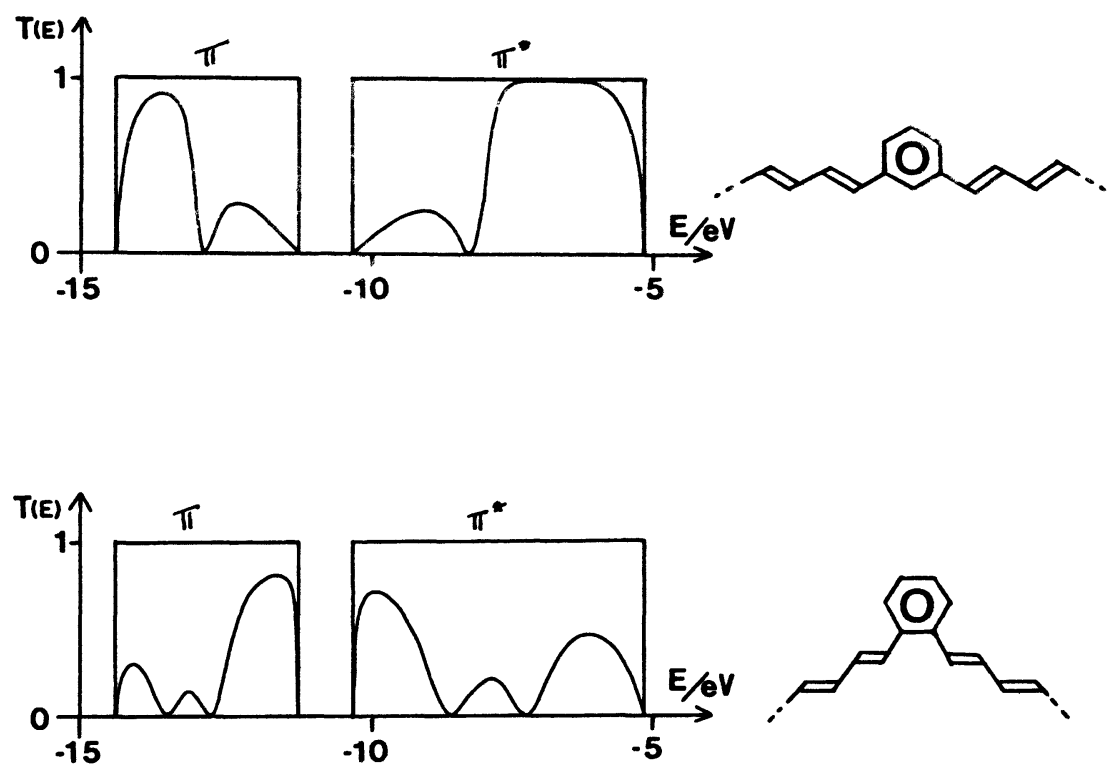

Fig. 5. - Transmission coefficient through a benzene ring embedded in a polyacetylene chain. The $t_{\alpha \alpha}^{2}$ coefficient are calculated from the $\pi$ and $\pi^{*}$ channel of a dimerized polyacetylene chain [34]. Notice the différence between the ortho and the meta connection.

[31] and not at all with absorbed molecules. In this last case, important for the characterization of the circuit figure 1, one can recognize the symmetry of a molecule on its chemisorbed molecule STM image [32]. But the detailed position of the atoms is not provided by such an approximation. More calculations are needed to take into account the atomic structure of the STM tip and the molecular orbital structure of the adsorbate [33]. We clearly need such a recognition step in our case.

\section{Conclusion.}

Molecular electronics taken in its strict meaning requires to work at the nanoscopic scale, molecule by molecule. Our experimental bench is an insulating surface where mesoscopic metallic wires have been fabricated. Therefore, the knowledge of inorganic surface atomic level structure is needed to be sure of the quality of the wiring and of the presence of the molecule. This will open the fascinating possibility to experiment on one (and the same) molecule and in the future to optimize the design of these molecules to get a specific fonction like a wire or a molecular switch.

\section{References}

[1] Simmons J.G., J. Appl. Phys 34 (1963) 1793.

[2] Fisher J.C. and GiaeVER I., J. Appl. Phys. 32 (1961) 172.

[3] Tsu R. and EsaKI L., Appl. Phys. Lett. 22 (1973) 562.

[4] Mann B. and Kunn H., J. Appl. Phys. 42 (1971) 4398. 
[5] Polymeropoulos E.E., J. Appl. 48 (1977) 2404.

[6] Aviram A. and Ratner M.A., Chem. Phys. Lett. 29 (1974) 277.

[7] MIKKELSEN K.V. and RATNER M.A., Chem. Rev. 87 (1987) 113.

[8] See for example: Radiationless processes in molecules and condensed phases. F.K. Fong Ed. (Springer Verlag. Berlin, 1976).

[9] CARTER F.L., Physica D 10 (1984) 175;

JOACHIM C. and LAUNAY J.P., New J. Chem. 12 (1984) 723;

LEHN J.M., Ang. Chem. Int. ed. 27 (1988) 89.

[10] AVIRAM A., JACS 110 (1988) 5687.

JOACHIM C. and LAUNAY J.P., J. Mol. Elec. 6 (1990) 37.

[11] JOACHIM C., New J. Chem., in press (1991).

[12] FRENKEL J., Phys. Rev. 36 (1930) 1604.

[13] Bloembergen N., Non Linear Optics, Frontiers in Physics (Benjamin, New York, 1965).

[14] SCHMid H., Rishton S.A., KERN D.P., WAShbURN S., WEBb R.A., KleinSASSER A., Chang T.H.P. and Fowler A., J. Vac. Sci. Techno. B. 6 (1988) 122.

[15] Datta S. and mc Lennan M.J., Rep. Prog. Phys. 53 (1990) 1003.

[16] ANDERson P.W., Thouless D.J., ABrahams E. and Fisher D.S., Phys. Rev. B. 22 (1980) 3519; STONE A.D. and SZAFER A., IBM J. Res. Dev. 32 (1988) 3841.

[17] See for example: VAN WEes B.J., VAN HoUTEN H., BEENAKKeR C.W.J., WilliaMSON J.G., KANWENHOVEn L.P., VAN DER MAREL D. and FoXon C.T., Phys. Rev. Lett. 60 (1988) 848.

[18] Landauer R., J. Phys. Cond. Matter. 1 (1989) 8099.

[19] BUTtTIKeR M., IMRY Y., LANDAueR R. and Pinhas S., Phys. Rev. B. 31 (1985) 6207.

[20] JOACHIM C., Ph. D. dissertation, Toulouse (1990).

[21] IMRY Y., in "Direction in condensed Matter Physics", Memorial volume in Honor of Shang-Keng Ma, G. Grinstein and G. Mazenko Eds. (World Scientific, Singapore, 1986) p. 101.

[22] JOACHIM C. and SAUTET P., Proceeding of the Solid State Science School on STM, Nato ARM, Erice 1989 (Springer Verlab, Berlin 1990) p.377.

[23] JOACHIM C., J. Mol. Electronic. 4 (1988) 125.

[24] JOACHIM C., LaunaY J.P. and WOITELlier S., Chem. Phys. 147 (1990) 131.

[25] Michel B., Travaglini G., Rohrer H., JoAChim C. and AmRein M., Z. Phys. B. 76 (1989) 99.

[26 DURIg U., JOACHIM C., HAUSSLING L. and MiCHEL B., in preparation.

[27] LAUnAY J.P., SOWINSKa M., LEYdier L., Gourdon A., AMOUYAl E., Bolllot M.L., Heisel F. and MIEHÉ J.A., Chem. Phys. Lett. 160 (1989) 89.

[28] JOACHIM C. and SAUTET P., Unpublished results.

[29] JOACHIM C., ROUSSET B., SCHÖNENBERGER C., KERRIEN A. and DRUET E., Nanotech. J., submitted (1991).

[30] TeRSOFF J. and Hamann D.R., Phys. Rev. Lett. 50 (1983) 1998;

TERSOFF J. and HAMANn D.R., Phys. Rev. Lett. 31 (1985) 805.

[31] CHEN C.J., Phys. Rev. Lett. 65 (1990) 448.

[32] Ohtani H., Wilson R.J., Chiang S. and Mate C.M., Phys. Rev. Lett. 60 (1988) 2398.

[33] SAUTET P. and JOACHIM C., Chem. Phys. Lett., in preparation (1991).

[34] SaUtet P. and Joachim C., Chem. Phys. Lett. 153 (1988) 511. 